\title{
The dyspnoea-inactivity vicious circle in COPD: development and external validation of a conceptual model
}

\author{
Maria A. Ramon (10 ${ }^{1,2}$, Gerben Ter Riet ${ }^{3}$, Anne-Elie Carsin $4,5,6,7$ \\ Elena Gimeno-Santos (10,8,9, Alvar Agustí ${ }^{2,8}$, Josep M. Antó ${ }^{4,5,6,7}$, \\ David Donaire-Gonzalez $\mathbb{1}^{4,5,6}$, Jaume Ferrer ${ }^{1,2,10}$, Esther Rodríguez ${ }^{1,2}$ \\ Robert Rodriguez-Roisin ${ }^{2,8}$, Milo A. Puhan ${ }^{11}$, Judith Garcia-Aymerich (10,5,6 and \\ the PAC-COPD Study Group
}

Affiliations: ${ }^{1}$ Dept of Pneumology, Hospital Universitari Vall d'Hebron, Barcelona, Spain. ${ }^{2}$ CIBER de Enfermedades Respiratorias (CIBERES), Barcelona, Spain. ${ }^{3}$ Dept of General Practice, Academic Medical Center, University of Amsterdam, Amsterdam, The Netherlands. ${ }^{4}$ ISGlobal, Barcelona, Spain. ${ }^{5} \mathrm{CIBER}$

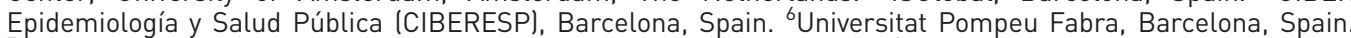
${ }^{7}$ IMIM (Hospital del Mar Medical Research Institute), Barcelona, Spain. ${ }^{8}$ Respiratory Institute, Hospital Clinic, Universitat de Barcelona, Barcelona, Spain. ${ }^{9}$ Institut d'Investigacions Biomèdiques August Pi i Sunyer, Barcelona, Spain. ${ }^{10}$ Dept of Medicine, Universitat Autònoma de Barcelona, Barcelona, Spain. ${ }^{11}$ Epidemiology, Biostatistics and Prevention Institute, University of Zurich, Zurich, Switzerland.

Correspondence: Judith Garcia-Aymerich, ISGlobal, Centre for Research in Environmental Epidemiology (CREAL), Dr Aiguader 88, 08003 Barcelona, Spain. E-mail: judith.garciađisglobal.org

@ERSpublications

An externally validated model highlights exercise capacity and exacerbations as drivers of the COPD vicious circle http://ow.ly/NXvu30kW7CP

Cite this article as: Ramon MA, Ter Riet G, Carsin A-E, et al. The dyspnoea-inactivity vicious circle in COPD: development and external validation of a conceptual model. Eur Respir J 2018; 52: 1800079 [https://doi.org/10.1183/13993003.00079-2018].

ABSTRACT The vicious circle of dyspnoea-inactivity has been proposed, but never validated empirically, to explain the clinical course of chronic obstructive pulmonary disease (COPD). We aimed to develop and validate externally a comprehensive vicious circle model.

We utilised two methods. 1) Identification and validation of all published vicious circle models by a systematic literature search and fitting structural equation models to longitudinal data from the Spanish PACCOPD (Phenotype and Course of COPD) cohort ( $n=210$, mean age 68 years, mean forced expiratory volume in $1 \mathrm{~s}$ (FEV1) $54 \%$ predicted), testing both the hypothesised relationships between variables in the model ("paths") and model fit. 2) Development of a new model and external validation using longitudinal data from the Swiss and Dutch ICE COLD ERIC (International Collaborative Effort on Chronic Obstructive Lung Disease: Exacerbation Risk Index Cohorts) cohort ( $n=226$, mean age 66 years, mean FEV1 57\% predicted).

We identified nine vicious circle models for which structural equation models confirmed most hypothesised paths but showed inappropriate fit. In the new model, airflow limitation, hyperinflation, dyspnoea, physical activity, exercise capacity and COPD exacerbations remained related to other variables and model fit was appropriate. Fitting it to ICE COLD ERIC, all paths were replicated and model fit was appropriate.

Previously published vicious circle models do not fully explain the vicious circle concept. We developed and externally validated a new comprehensive model that gives a more relevant role to exercise capacity and COPD exacerbations. 


\section{Introduction}

Chronic obstructive pulmonary disease (COPD) is characterised by chronic airflow limitation and persistent respiratory symptoms that limit patients' activities [1, 2]. The reduction in physical activity [3] leads to patients' physical deconditioning and further impairment of respiratory symptoms [4]. This process is known as the "disease spiral" or "vicious circle" theory of dyspnoea-inactivity in COPD [5], a construct that has been helpful so far in understanding patients' concerns and generating research hypotheses $[3,6]$. However, the literature contains several versions of the vicious circle, likely because they are based on expert opinion, clinical observations and associations reported in cross-sectional studies only [5, 7-9]. Furthermore, the vicious circle theory in COPD has never been validated prospectively.

We argue that the dyspnoea-inactivity vicious circle can be conceived as a "conceptual model", i.e. a set of direct and indirect relationships among variables involved in a specific health problem usually represented by a diagram [10]. In order to build such a model, it is important to establish which are the variables and the relations between variables ("paths") that play a part in the dyspnoea-inactivity vicious circle. Therefore, we aimed to establish an empirically validated model for the dyspnoea-inactivity vicious circle in COPD through: 1) identification and validation of all published vicious circle models using real patients' longitudinal measurements, and 2) building and external validation of a comprehensive new vicious circle model, using repeated measurements from two European COPD cohort studies.

\section{Methods}

Systematic literature review

We conducted a systematic literature review to identify all previously published conceptual models for the dyspnoea-inactivity vicious circle in COPD following the guidelines of the Centre for Reviews and Dissemination [11], the Cochrane Collaboration [12], and the PRISMA statement for reporting of systematic reviews [13]. All methods were specified in advance and documented in a protocol (see supplementary material). Briefly, we searched PubMed/MEDLINE and SCOPUS databases from the earliest to the most recent May 2017 records (see full search strategy in the supplementary material). Articles were included if they discussed or explained the dyspnoea-inactivity vicious circle in a diagram. No date or language restrictions were imposed. Two of the co-authors (M.A. Ramon and E. Gimeno-Santos) independently reviewed the title and abstract of every citation retrieved by the database searches, and a third co-author (J. Garcia-Aymerich) decided upon inclusion in case of disagreement. For each study we rebuilt its vicious circle diagram in the form of a directed acyclic graph depicting the hypothesised longitudinal relationships (both direct and indirect) between involved variables. To account for the cyclic nature of relationships between variables involved in most vicious circle models, we considered most variables as time varying and included several time-points (e.g. dyspnoea at $t 1 \rightarrow$ physical activity at $t 2 \rightarrow$ dyspnoea at $t 3$ ). A representative of each original paper (first or corresponding author) was contacted and all (except one, who did not respond to several mail requests) agreed with our adaptation of their diagram.

\section{Study design and participants of the European COPD cohort studies}

We had access to individual patient data of two COPD multicentre cohort studies that measured longitudinally variables potentially involved in the dyspnoea-inactivity vicious circle. 1) The PAC-COPD (Phenotype and Course of COPD) project that recruited patients during their first COPD exacerbation in eight hospitals in Spain $(\mathrm{n}=342)$ and evaluated their characteristics during clinical stable conditions every 12-18 months, up to a maximum of three times [14]. 2) The ICE COLD ERIC (International Collaborative Effort on Chronic Obstructive Lung Disease: Exacerbation Risk Index Cohorts) study that recruited patients in primary care centres from the Netherlands and Switzerland $(n=409)$ and evaluated them every 6 months up to 5 years [15]; for the present study we included data from every 2 years up to three times, corresponding to the time span available in PAC-COPD cohort. A total of 210 and 226 patients from the PAC-COPD and the ICE COLD ERIC cohorts, respectively, had dyspnoea and physical activity data available at the three consecutive study time-points, and were therefore included in the present analyses (supplementary tables S1 and S2). All included patients had spirometry-defined COPD (post-bronchodilator forced expiratory volume in $1 \mathrm{~s}\left(\mathrm{FEV}_{1}\right) /$ forced vital capacity ratio $<0.70$ during clinical stable conditions). The two cohort studies were approved by the relevant ethics committees and written informed consent was obtained from each participant.

\section{Measurements}

All variables involved in at least one of the vicious circle models identified in the systematic literature review had available repeated measurements in the PAC-COPD cohort and most of them in the ICE COLD ERIC cohort. These included the following. 1) The modified Medical Research Council (mMRC) dyspnoea scale (score 0-4) in both cohorts, which grades breathlessness during daily activities [16]. 2) Physical activity by the estimated weekly energy expenditure $\left(\mathrm{kcal}^{\mathrm{w}} \mathrm{week}^{-1}\right)$ using the Yale Physical Activity 
Survey (YPAS) $[17,18]$ in the PAC-COPD cohort and the physical activity total score (0-23) from the Longitudinal Aging Study Amsterdam Physical Activity Questionnaire (LAPAQ) [19] in the ICE COLD ERIC cohort. Both YPAS and LAPAQ questionnaires ask about the frequency, intensity and duration of a list of activities not related to dyspnoea or other respiratory symptoms, in the previous month and previous 2 weeks, respectively. The YPAS questionnaire allows for the calculation of weekly energy expenditure in physical activity, while the LAPAQ questionnaire assigns weights to each activity according to the metabolic equivalent task, giving a score that ranges from 0 to 23. 3) The 6-min walk distance and both the maximal ventilation during the incremental test and lactic acid from a cardiopulmonary incremental exercise test with a cycloergometer in the PAC-COPD study, and the number of repetitions in the 1-min sit-to-stand test in the ICE COLD ERIC study [20]. 4) Handgrip force in both studies. 5) FEV1, before and after bronchodilation, and inspiratory capacity (IC) by spirometry in both studies; total lung capacity (TLC), diffusing capacity of the lung for carbon monoxide and arterial oxygen tension. IC and IC/TLC were taken as indices of inspiratory constraint derived from static hyperinflation of the lung [21]. 6) Charlson index of comorbidities, only available in the PAC-COPD. 7) COPD exacerbations gathered from governmental databases in the PAC-COPD study, and through central adjudication of both patient interviews and review of patient records in the ICE COLD ERIC study. 8) Health-related quality of life by the St George's Respiratory Questionnaire total score (0-100) in the PAC-COPD study and the Chronic Respiratory Questionnaire total score in the ICE COLD ERIC study. 9) The Hospital Anxiety and Depression Scale scores in both cohorts.

Detailed information about the methods, questionnaires, standardisation of the tests and fieldwork supervision of the two studies has been reported previously [22, 23].

\section{Statistical analysis}

Sample size allowed a statistical power $>99 \%$ to identify as statistically significant a better fit of our model than previously published models (see power calculations in the supplementary material) [24].

We appraised the validity of vicious circle models obtained from the systematic review (first objective) by fitting a structural equation model (SEM) with the variables and paths depicted in each directed acyclic graph (corresponding to each identified vicious circle model) using longitudinal data from the PAC-COPD cohort and testing 1) the hypothesised relationships between variables ("paths") and 2) how well the full hypothesised model fitted the data. Briefly, a SEM is a statistical modelling technique that tests a construct of data (including variables and paths) to estimate direct and indirect relationships between the included variables [25]. First, the path coefficients estimated by the SEM informed about each hypothesised path in the model. Path coefficients were standardised ranging from -1 to +1 to facilitate comparison between paths involving different variables and can be interpreted as correlation coefficients [25]. Nonstandardised coefficients were also used to better understand the strength of associations between variables in the model and to be able to compare them with previous research. Statistically significant path coefficients in the hypothesised direction (e.g. dyspnoea negatively associated with later physical activity) supported validity of the model. Second, model fit parameters informed about how well the variables and paths included in a specific model actually represented the underlying concept (the dyspnoea-inactivity vicious circle) [26].

The three following SEM fit criteria were considered [27-31]: 1) Chi-squared/degrees of freedom (d.f.) $<2.0$ and a nonsignificant $(\geqslant 0.05) \mathrm{p}$-value, which indicates that a nonsignificant amount of variance in the data remains unexplained $[27,30,31], 2)$ a root mean square error of approximation (RMSEA) $<0.07$ with the $90 \%$ CI between 0.05 and 0.10 , which indicates that the model fits well with the population covariance matrix $[28,30,31]$, and 3 ) a comparative fit index (CFI) $\geqslant 0.95$, which indicates that variables included in the model are well correlated among them $[29,31]$. A model had to fulfil all our three criteria. If not, we considered it to not appropriately explain the vicious circle theory (e.g. because the model missed some variables or paths relevant to the vicious circle).

We decided a priori to propose a new vicious circle model if none of the models identified in the systematic literature review proved to be valid. As this was the case (see Results section), we built a new vicious circle model (second objective), including as candidate variables all variables and paths involved in at least one of the previously identified vicious circles, by fitting a new SEM to PAC-COPD longitudinal data. In addition, we allowed the SEM to add any path (missing in previous models) that was statistically significant and improved the model fit, only if it was biologically plausible (e.g. a direct path between airflow limitation and dyspnoea). In an iterative process of removing paths or variables one at a time, we removed from the SEM 1) paths with not statistically significant path coefficients $(p \geqslant 0.05)$ and 2$)$ variables not related to any other variable in the model (path coefficients with $p \geqslant 0.05$ ) whose removal did not worsen the model fit. The final SEM kept variables that showed a statistically significant association with at least one of the remaining variables and/or significantly improved the model fit. As a final step, we fitted the final SEM model obtained with the PAC-COPD cohort to the ICE COLD ERIC longitudinal 
data. The model was considered a valid representation of the vicious circle if it met our criteria detailed above (confirmation of paths and appropriate model fit).

All analyses were performed with Stata version 12.1 (StataCorp, College Station, TX, USA).

\section{Results}

Identification of vicious circle models in the systematic literature review

We identified nine articles that reported the conceptual model of the dyspnoea-inactivity vicious circle as a diagram (supplementary figure S1) [5, 7, 8, 32-37]. There were differences in variables and paths across different models (table 1 and figure 1). Dyspnoea was included in all models, exercise capacity in eight models, physical activity in seven models and muscle strength in six models. Most of the models (six out of nine) included dyspnoea as the starting variable of the vicious circle, whereas airflow limitation was considered the initial factor in the remaining three models.

For each of the identified diagrams we built a directed acyclic graph (figure 1).

\section{Validation of the vicious circle models identified in the systematic literature review}

We fitted a SEM for each of the identified nine diagrams to the PAC-COPD longitudinal data. Patients were mostly male (93\%), had mean FEV1 54\% predicted and median mMRC dyspnoea score of 2 (table 2) [38]. Dyspnoea, physical activity, and other clinical and functional variables deteriorated during follow-up (supplementary table S3).

Most paths (of the nine models) were replicated with statistically significant path coefficients in the hypothesised direction (figure 1). However, none of the vicious circle models showed appropriate fit as per p-values (all <0.05), RMSEA values $(0.176-0.352)$ and CFI values $(0.347-0.629)$ (table 3).

\section{Building a comprehensive new vicious circle model}

Supplementary figure S2 shows all variables and paths involved in at least one of the vicious circle models identified in the systematic review. After several iterations using PAC-COPD data we obtained a final model (figure 2a) in which 1) both airflow limitation and lung hyperinflation were directly associated with dyspnoea, 2) dyspnoea was related to future exercise capacity both directly and mediated by a reduction in physical activity, 3) both physical activity and exercise capacity were related to future dyspnoea levels, 4) COPD exacerbations, associated with prior airflow limitation, were related to future exercise capacity and dyspnoea, 5) other outcomes (e.g. quality of life, anxiety, depression, comorbidities, hypoxaemia or muscle weakness) did not contribute to the vicious circle once the abovementioned variables and paths were

$\begin{array}{lccc}\begin{array}{l}\text { TABLE 1 Reference details, type of article and list of variables of nine studies reporting the } \\ \text { dyspnoea-inactivity vicious circle in a diagram identified through the systematic literature } \\ \text { search }\end{array} & \text { Year } & \begin{array}{c}\text { Type of } \\ \text { article }\end{array} & \text { Variables involved in the dyspnoea-inactivity vicious circle } \\ \text { Reference } & 2001 & \text { Review } & \text { Dyspnoea, exercise capacity, muscle force, ventilatory } \\ \text { requirements }\end{array}$




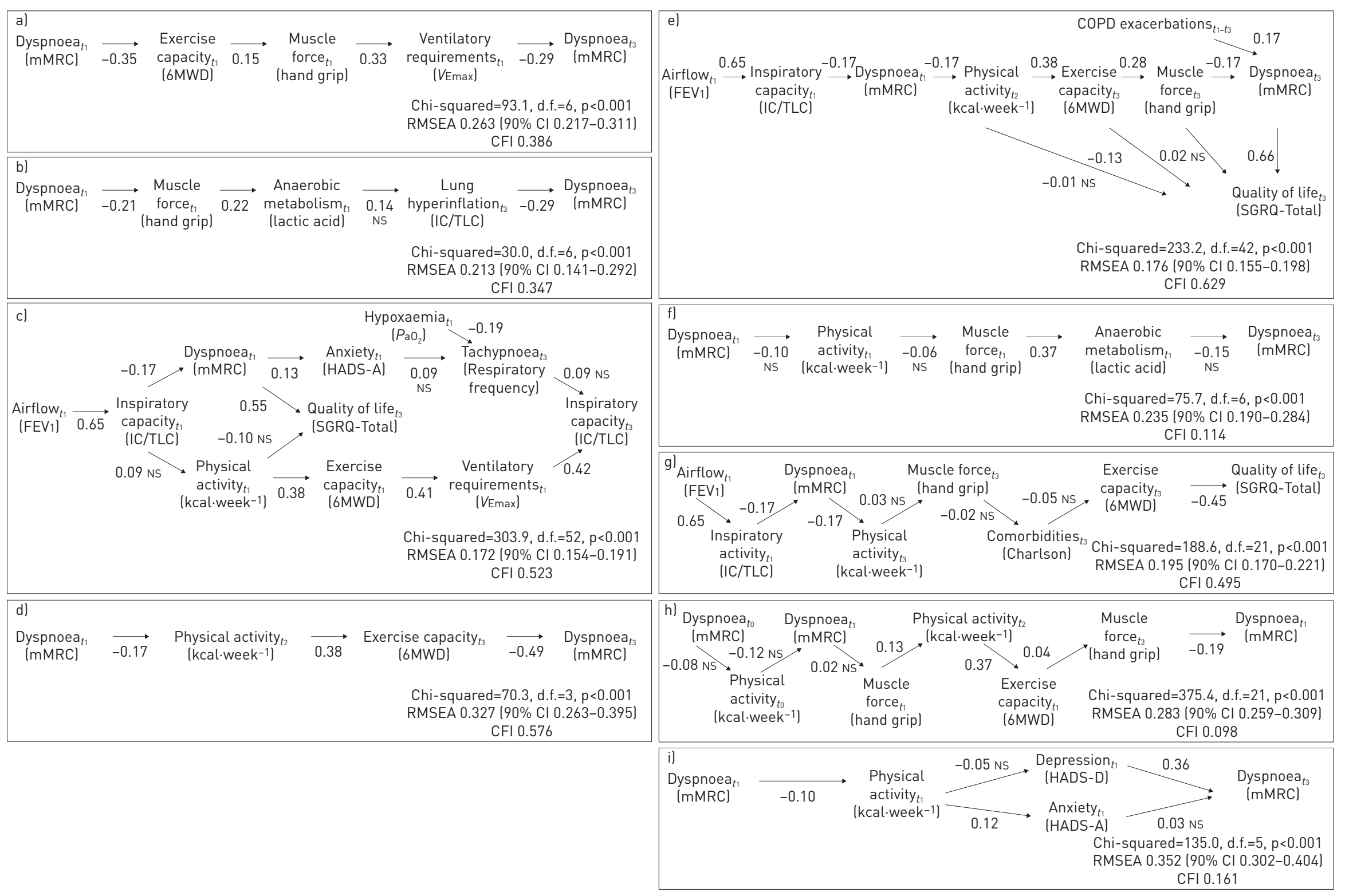

FIGURE 1 Directed acyclic graphs and validation\# of the identified diagrams for the dyspnoea-inactivity vicious circle in chronic obstructive pulmonary disease (COPD): models proposed by a) Cooper [5], b) Polkey and Moxhan [8], c) Cooper [32], d) Reardon et al. [7], el Decramer [33], f) Donaldson et al. [34], g) Maltals [35], h) Garcia-Armerich and Pitta [36], and i) CorhaY et al. [37]. mMRC: modified Medical Research Council; 6MWD: 6-min walk distance; VEmax: maximal ventilation during incremental cycloergometer test; IC: inspiratory capacity; TLC: total lung capacity; FEV1: forced expiratory volume in $1 \mathrm{~s}$; HADS-A/D: Hospital Anxiety and Depression Scale-Anxiety/Depression; SGRQ: St George's Respiratory Questionnaire (higher scores indicate more limitations); $\mathrm{PaO}_{2}$ : arterial oxygen tension; d.f.: degrees of freedom; RMSEA: root mean square error of approximation; CFI: comparative fit index; NS: nonsignificant. \#: using standardised coefficients the magnitude of the association between two variables takes values ranging from -1 to +1 , where negative values indicate a negative relationship. 
TABLE 2 Patients' characteristics in the PAC-COPD (Phenotype and Course of Chronic Obstructive Pulmonary Disease) and ICE COLD ERIC (International Collaborative Effort on Chronic Obstructive Lung Disease: Exacerbation Risk Index Cohorts) cohorts at recruitment

\begin{tabular}{|c|c|c|}
\hline & PAC-COPD & ICE COLD ERIC \\
\hline Patients & 210 & 226 \\
\hline \multicolumn{3}{|l|}{ Anthropometric and clinical data } \\
\hline Male & 195 (92.9) & $135(60.0)$ \\
\hline Age years & $67.5 \pm 8.2$ & $65.7 \pm 9.5$ \\
\hline Active smoker & $74(35.2)$ & 80 (35.4) \\
\hline Physical activity YPAS scale kcal.week ${ }^{-1}$ & 6056 (3345-9085) & \\
\hline Physical activity LAPAQ-Total score & & $13(9-15)$ \\
\hline SGRQ-Total score & $31.2(22.2-44.1)$ & \\
\hline CRQ score (mean of four domains) & & $5.2(4.4-6.0)$ \\
\hline HADS-Anxiety score & $4(2-7)$ & $4(2-7)$ \\
\hline HADS-Depression score & $3(1-5)$ & $3(2-6)$ \\
\hline Charlson index of comorbidity & $2(1-2)$ & \\
\hline mMRC dyspnoea score & $2(2-3)$ & $1(1-3)$ \\
\hline Respiratory frequency breaths $\cdot \min ^{-1}$ & $18(16-20)$ & \\
\hline \multicolumn{3}{|l|}{ Lung function } \\
\hline Post-bronchodilator FEV1 \% pred & $53.5 \pm 16.6$ & $56.6 \pm 16.9$ \\
\hline \multicolumn{3}{|l|}{ Airflow limitation severity ${ }^{\#}$} \\
\hline Mild (FEV $1 \geqslant 80 \%)$ & $13(6.2)$ & $10(4.4)$ \\
\hline Moderate (FEV1 50-<80\%) & $104(49.5)$ & 139 (61.5) \\
\hline Severe (FEV $130-<50 \%)$ & $79(37.6)$ & $61(27.0)$ \\
\hline Very severe (FEV $1<30 \%$ ) & $14(6.7)$ & $16(7.1)$ \\
\hline IC/TLC \% & $31.4 \pm 0.9$ & \\
\hline IC \% pred & $69.5 \pm 20.7$ & $73.9 \pm 20.8$ \\
\hline $\mathrm{PaO}_{2} \mathrm{mmHg}$ & $74.8 \pm 11.3$ & \\
\hline \multicolumn{3}{|l|}{ Exercise capacity and muscle force } \\
\hline 6MWD m & $445 \pm 84$ & \\
\hline Sit-to-stand repetitions $\mathrm{n}$ & & $19.9 \pm 9.7$ \\
\hline$V_{\text {Emax }} L \cdot \min ^{-1}$ & $42.2 \pm 12.7$ & \\
\hline Lactic acid mM & $4.8 \pm 2.2$ & \\
\hline Handgrip muscle force $\mathrm{kg}$ & $31.4 \pm 8.2$ & $31.7 \pm 11.6$ \\
\hline \multicolumn{3}{|c|}{$\begin{array}{l}\text { Data are presented as } n, n(\%) \text {, mean } \pm S D \text { or median (interquartile range). YPAS: Yale Physical Activity } \\
\text { Survey; LAPAQ: Longitudinal Aging Study Amsterdam Physical Activity Questionnaire; SGRQ: St George's } \\
\text { Respiratory Questionnaire; CRQ: Chronic Respiratory Questionnaire; HADS: Hospital Anxiety and } \\
\text { Depression Scale; mMRC: modified Medical Research Council; FEV1: forced expiratory volume in } 1 \mathrm{~s} \text {; IC: } \\
\text { inspiratory capacity; TLC: total lung capacity; } \mathrm{PaO}_{2} \text { : arterial oxygen tension; } 6 \mathrm{MWD:} 6 \text {-min walk distance; } \\
\text { VEmax: maximal ventilation during incremental cycloergometer test. " } \text { : according to the criteria of the } \\
\text { American Thoracic Society/European Respiratory Society [38]. }\end{array}$} \\
\hline
\end{tabular}

included in the model, and 6) model fit was good according to all parameters (Chi-squared=5.8, d.f. $=8$, $\mathrm{p}=0.667$, RMSEA $<0.001(90 \%$ CI $<0.001-0.058)$ and CFI $>0.999)$. Supplementary figure S3a shows this SEM model using nonstandardised coefficients.

Validation of the new vicious circle model

To validate this new model, we applied it to the ICE COLD ERIC longitudinal data. These patients $(60 \%$ males, mean FEV1 57\% predicted and median mMRC dyspnoea score of 1) had in general milder COPD than those of PAC-COPD (table 2), but their clinical and functional characteristics also deteriorated during follow-up (supplementary table S4). Figure $2 \mathrm{~b}$ shows results of the fitted SEM, which confirmed all paths with statistically significant path coefficients in the hypothesised direction and showed a good fit to the data according to all parameters (Chi-squared=14.6, d.f. $=8, p=0.067$, RMSEA 0.060 (90\% CI $<0.001-$ 0.101) and CFI 0.984). Supplementary figure S3b shows this SEM model using nonstandardised coefficients. Finally, figure 3 shows a diagram depicting the new vicious circle conceptual model.

\section{Discussion}

To the best of our knowledge, this is the first study that developed and externally validated a model for the dyspnoea-inactivity vicious circle theory in COPD. We found that 1) previously published conceptual models contain valid information about the vicious circle (as most paths could be replicated using 
TABLE 3 Validity appraisal of the nine vicious circle models identified in the systematic literature search

\begin{tabular}{|c|c|c|c|c|}
\hline & \multirow{2}{*}{$\begin{array}{l}\text { Paths confirmation } \\
\text { [confirmed paths }{ }^{\# / \text { total }} \\
\text { hypothesised paths) }\end{array}$} & \multicolumn{3}{|c|}{ Model fit } \\
\hline & & $\begin{array}{c}\text { Chi-squared, d.f., } \\
\text { p-value }\end{array}$ & RMSEA $(90 \% \mathrm{Cl})$ & CFI \\
\hline Threshold value & & $\begin{array}{l}\text { Chi-squared/d.f. } \\
<2.0 \text { and } p \geqslant 0.050\end{array}$ & $<0.070(0.050-0.100)$ & $>0.950$ \\
\hline \multicolumn{5}{|l|}{ Reference } \\
\hline COOPER [5] & $4 / 4$ & $93.1,6,<0.001$ & $0.263(0.272-0.390)$ & 0.138 \\
\hline PoLkEY and Moxhan [8] & $3 / 4$ & $30.0,6,<0.001$ & $0.213(0.141-0.292)$ & 0.347 \\
\hline COOPER [32] & $8 / 12$ & $303.9,52,<0.001$ & $0.172(0.154-0.191)$ & 0.523 \\
\hline ReARDon et al. [7] & $3 / 3$ & $70.3,3,<0.001$ & $0.327(0.263-0.395)$ & 0.576 \\
\hline DeCramer [33] & $9 / 11$ & $233.2,42,<0.001$ & $0.176(0.155-0.198)$ & 0.629 \\
\hline Donaldson et al. [34] & $1 / 4$ & $75.7,6,<0.001$ & $0.235(0.190-0.284)$ & 0.114 \\
\hline MaLtaIs [35] & $4 / 7$ & $188.6,21,<0.001$ & $0.195(0.170-0.221)$ & 0.495 \\
\hline Garcia-Aymerich and PITta [36] & $4 / 7$ & $375.4,21,<0.001$ & $0.283(0.259-0.309)$ & 0.098 \\
\hline CoRHAY et al. [37] & $3 / 5$ & $135.0,5,<0.001$ & $0.352(0.302-0.404)$ & 0.161 \\
\hline
\end{tabular}

longitudinal data), but do not likely explain the vicious circle concept appropriately (as per the low fit of the models to patients' data), and 2) a new comprehensive model that gives a more relevant role to exercise capacity and COPD exacerbations was built and validated using two prospective cohorts including COPD patients with different degrees of disease severity from different geographic areas and clinical settings.

\section{Interpretation of findings}

We found that existing vicious circle models included variables and relations between variables ("paths") relevant to the vicious circle. These variables and paths describe a sequence of events from expiratory airflow limitation, increase in resting lung volumes and dynamic hyperinflation, to a shallow and rapid breathing pattern with worsening dyspnoea, decrease in physical activity and a deterioration of exercise capacity, which further enhances dyspnoea, thus akin to the current knowledge on the pathophysiology of COPD $[39,40]$.

However, we observed that existing vicious circle models show a poor fit to real patients' longitudinal data. Our findings suggest that previous models did not fully represent the underlying theory (i.e. the dyspnoea-inactivity vicious circle) because additional variables and paths should have been considered. The acquisition of good fit indices when the model built with PAC-COPD data was used on the ICE COLD ERIC data supports this assumption. Our results also suggest that any adaptation of the current vicious circle model to other chronic respiratory conditions would require real patients' data rather than simple extrapolation from COPD.

The new vicious circle model that we propose has two main differences compared with previous models. First, we found that COPD exacerbations, key events in the natural history of COPD and only considered in one previous model [33], are of relevance to the vicious circle, even in a primary care COPD population with infrequent exacerbations like ICE COLD ERIC. Moreover, our model supported a bidirectional role for exacerbations (both affecting future variables of the vicious circle, as previously suggested [33], and also affected by prior variables), consistent with the current view that attributes a prominent role to exacerbations in COPD assessment, management and prognosis [1]. Second, our results show that exercise capacity has a more central role in the vicious circle than previously considered [5, 32-35], given that most of the effects of other variables (lung hyperinflation, physical activity, exacerbations) on dyspnoea are mediated by effects on exercise capacity. Again, this finding is consistent with existing knowledge on COPD [41, 42], as exercise capacity decline has been found to be accelerated by hyperinflation of the lung [41] and COPD exacerbations [42], and improving exercise capacity (e.g. through an exercise training programme [43]) reduces dyspnoea.

Several explanations may help to interpret why some variables included in previous vicious circle models and relevant to COPD prognosis, e.g. skeletal muscle weakness, anxiety or depression and comorbidities, did not remain in our final model $[32,34,35,37]$. 1) Patients with higher prevalence of these conditions 
a)

)

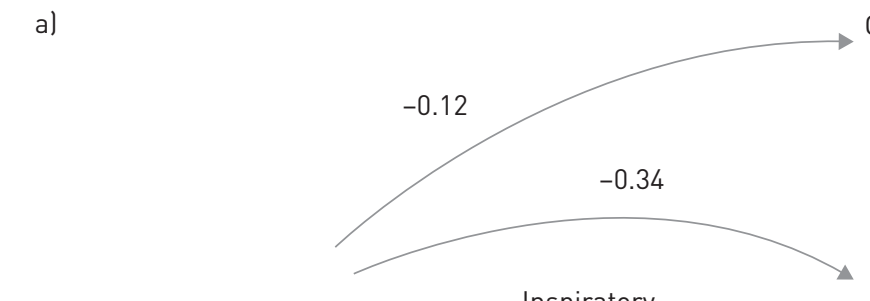

COPD exacerbations

COPD
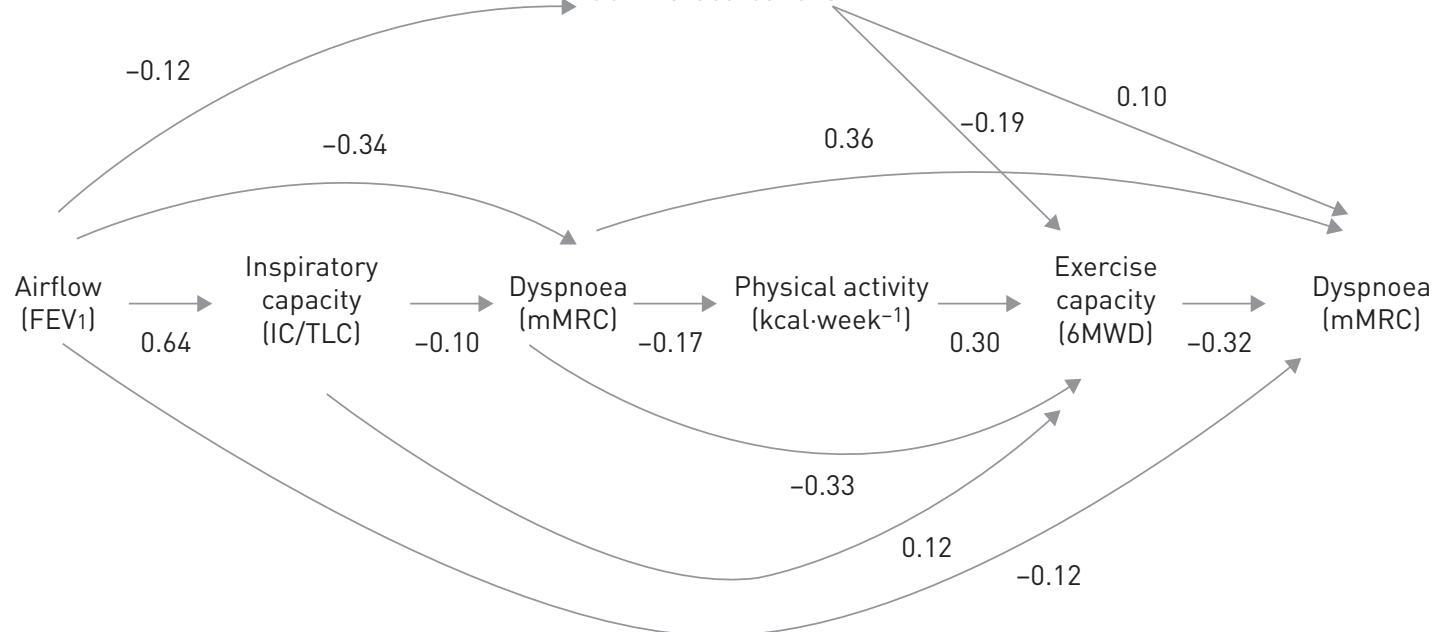

$-0.17$

(kcal-week ${ }^{-1}$ )

0.30

$-0.32$ ImMRC)

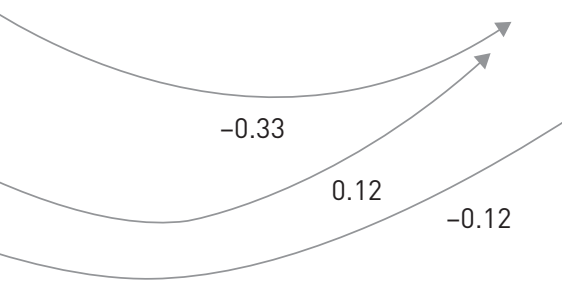

Chi-squared $=5.8, d . f .=8 ; p=0.667$

RMSEA $<0.001(90 \% \mathrm{Cl}<0.001-0.058)$

$\mathrm{CFI}>0.999$

b)

COPD exacerbations

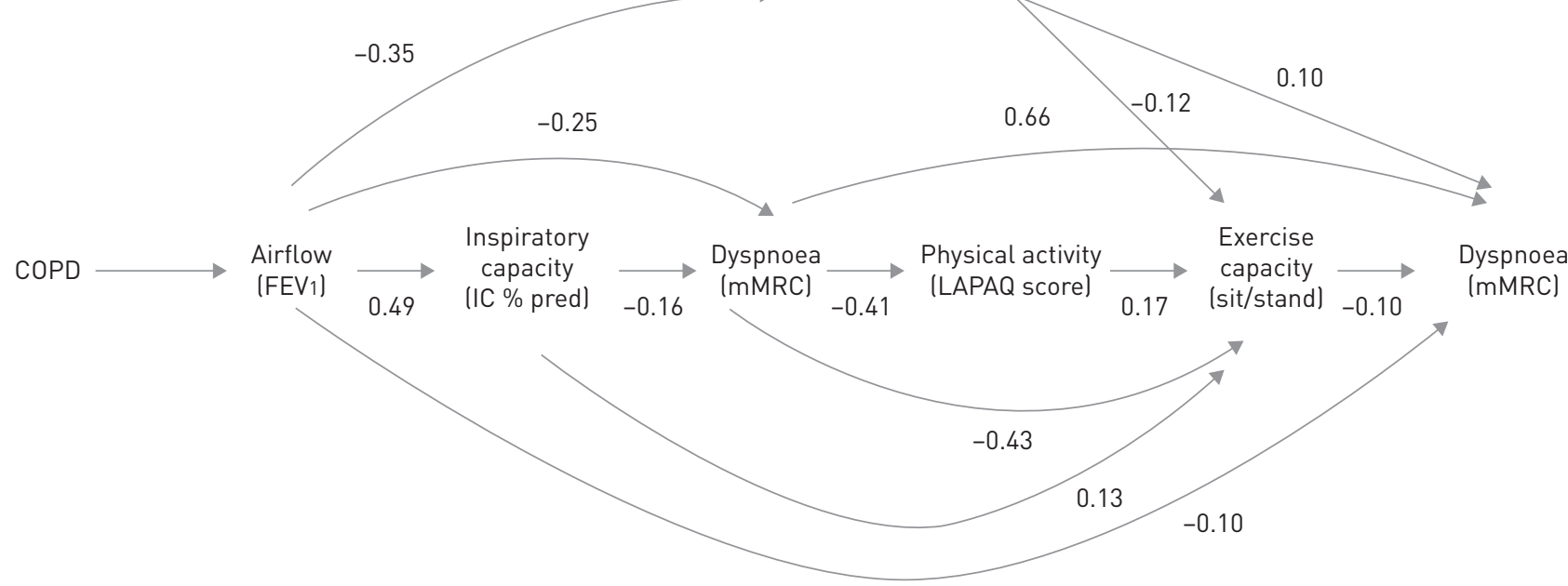

Chi-squared $=14.6$, d. $f .=8 ; p=0.067$ RMSEA 0.060 (90\% Cl<0.001-0.101) CFI 0.984

FIGURE 2 The new model of the dyspnoea-inactivity vicious circle in patients with chronic obstructive pulmonary disease (COPD): a) derivation and b) validation. Relationship and standardised path coefficients ${ }^{\#}$ between variables in the dyspnoea-inactivity vicious circle in patients with COPD. FEV1: forced expiratory volume in 1 s; IC: inspiratory capacity; TLC: total lung capacity; mMRC: modified Medical Research Council; 6MWD: 6-min walk distance; LAPAQ: Longitudinal Aging Study Amsterdam Physical Activity Questionnaire; d.f.: degrees of freedom; RMSEA: root mean square error of approximation; $\mathrm{CFI}$ : comparative fit index. " : using standardised coefficients the magnitude of the association between two variables takes values ranging from -1 to +1 , where negative values indicate a negative relationship.

were lost to follow-up, which could have prevented us from observing variability in important variables that could contribute to the vicious circle. 2) It is possible that, once other variables and paths were specified, those variables did not provide additional information to the vicious circle. 3) The available measures of these concepts in the PAC-COPD and ICE COLD ERIC cohorts may not have been sufficiently accurate. For example, our use of handgrip force instead of quadriceps force might have prevented us from finding stronger associations between skeletal muscle force and other variables in the models. 4) Variables important to COPD course, such as smoking history or body mass index, were not 


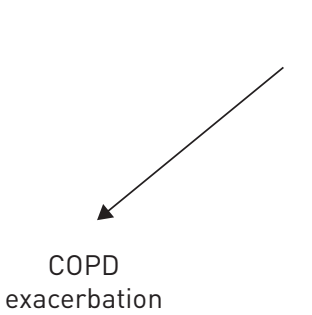

Airflow

limitation
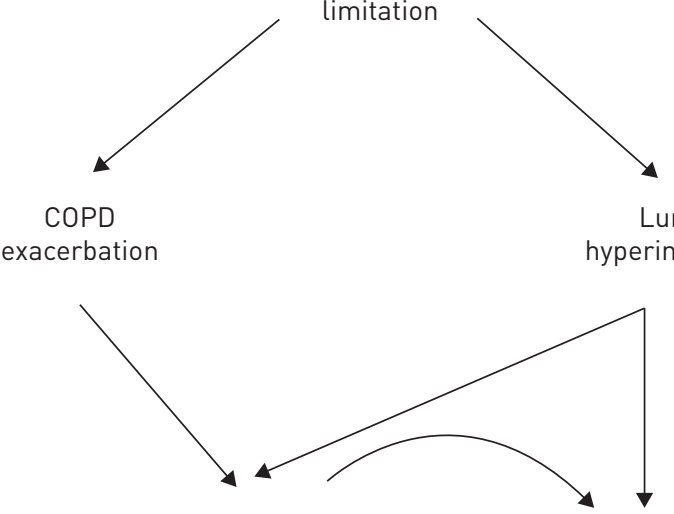

Exercise

limitation

Dyspnoea

FIGURE 3 Graphical representation of the new model of the dyspnoeainactivity vicious circle in patients with chronic obstructive pulmonary disease (COPD).
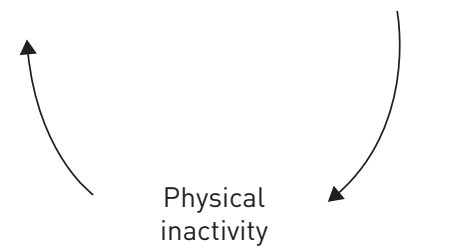

included in previous vicious circle models. Their inclusion could be considered after appropriate systematic reviews of how they affect and/or are affected by variables in the current vicious circle. Altogether, future research on this field will require repeated, valid measurements over time of all variables potentially related to the vicious circle phenomenon.

\section{Strengths and limitations}

A strength of our study is that we followed rigorous systematic review methodology to identify all previously published models for the dyspnoea-inactivity vicious circle. Also, we used SEMs to validate each of the identified models, which provides an extensive insight into the complex relationships between variables involved in the vicious circle. Finally, we used two different cohorts from different clinical settings (primary care and specialised hospital respiratory departments) including patients with a wide range of severity of their disease, which increases the external validity of our model.

Among potential limitations, first, the aforementioned lack of some relevant variables, e.g. quadriceps force, may have resulted in a relevant variable or path not being included in our final model. Second, the present article is based on a secondary analysis of two cohorts recruited with other primary research questions. As a consequence, some of the variables included in the analysis may not be the most appropriate for the study of the vicious circle concept. For example, the use of the mMRC scale (instead of other measures) to assess dyspnoea could make the argument of its association with physical activity a bit circular. Third, patients lost to follow-up in both cohorts had worse clinical and functional status at baseline, which may have reduced variability in some parameters, thus limiting the ability to identify associations. However, patients who present at the clinical practice with the typical manifestations of the vicious circle are actually those who survive to COPD without severe comorbidities, i.e. those who are followed-up in research studies. Accordingly, our findings cannot be extended to COPD patients with very limited survival or severe comorbidities. Fourth, a questionnaire was used to measure physical activity in both cohorts, which could be subject to poor accuracy at the individual level of a variable key to the vicious circle. (Of note, physical activity from an accelerometer was available in the second follow-up of the PAC-COPD study but not included in this analysis in order to keep the required temporal sequence.) Fifth, sample size of both cohorts was relatively small and did not allow testing any potential role of drug and nondrug treatments on the vicious circle. Sixth, although data were available for three different time-points ( $t_{1}, t_{2}$ and $t_{3}$ ), many variables were only available at $t_{1}$ and $t 3$, which may have hindered the identification of differences over time for some parameters. Furthermore, the relatively short follow-up period of our cohorts ( $<5$ years) could have limited the ability to identify novel variables relevant to the vicious circle or to estimate appropriately the contribution of relevant variables to the vicious circle. However, in both PAC-COPD and ICE COLD ERIC patients, we observed a statistically significant worsening over time in physical activity, lung function, exercise capacity and muscle force. Finally, the 
external validity of our new vicious circle model might not hold in the presence of large heterogeneity in COPD progression parameters.

\section{Conclusions}

Previously published vicious circle models do not fully explain the vicious circle concept. We developed and externally validated a new comprehensive model that gives a more relevant role to exercise capacity and COPD exacerbations. This new model may be of help to both clinicians and researchers to better understand the relationships among COPD characteristics involved in the dyspnoea-inactivity vicious circle, thus facilitating the design and testing of targeted therapeutic interventions.

Acknowledgements: We would like to thank the authors of the original articles for reviewing and accepting our proposal to validate their proposed model for the dyspnoea-inactivity vicious circle: Christopher Cooper (David Geffen School of Medicine, University of California, Los Angeles, CA, USA), Jean-Louis Corhay (Centre Hospitalier Universitaire, Sart-Tilman, Liège, Belgium), Marc Decramer (Respiratory Rehabilitation and Respiratory Division, University Hospital Leuven, Leuven, Belgium), François Maltais (Heart and Lung Institute, University of Laval, Quebec, QC, Canada), Michael Polkey (Dept of Respiratory Medicine, Royal Brompton Hospital, London, UK) and Richard ZuWallack (St Francis Hospital Medical Center, Hartford, CT, USA).

Conflict of interest: A. Agustí reports receiving grants and personal fees from AstraZeneca, GSK and Menarini, grants from MSD, and personal fees from Novartis, Teva and Chiesi, outside the submitted work. R. Rodriguez-Roisin reports receiving grants from Almirall and Menarini, personal fees for service on an advisory board from Boehringer Ingelheim, Pearl Therapeutics and Teva, and lecture fees from Novartis and Takeda, during the conduct of the study, all related to COPD. J. Garcia-Aymerich reports payments to her institution for consulting and lecturing from AstraZeneca, and receiving lecture fees from Esteve and Chiesi, outside the submitted work.

Support statement: The PAC-COPD study is funded by grants from Fondo de Investigación Sanitaria (FIS PI020541), Ministry of Health, Spain; Agència d'Avaluació de Tecnologia i Recerca Mèdiques (AATRM 035/20/02), Catalonia Government; Spanish Society of Pneumology and Thoracic Surgery (SEPAR 2002/137); Catalan Foundation of Pneumology (FUCAP 2003 Beca Marià Ravà); Red RESPIRA (RTIC C03/11); Red RCESP (RTIC C03/09), Fondo de Investigación Sanitaria (PI052486); Fondo de Investigación Sanitaria (PI052302); Fundació La Marató de TV3 (041110); DURSI (2005SGR00392); and unrestricted educational grants from Novartis Farmacéutica, Spain, and AstraZeneca Farmacéutica, Spain. CIBERESP and CIBERES are funded by the Instituto de Salud Carlos III, Ministry of Health, Spain. ISGlobal is a member of the CERCA Programme, Generalitat de Catalunya. The ICE COLD ERIC COHORT study was funded by the Swiss National Science Foundation (grant 3233B0/115216/1), Dutch Asthma Foundation (grant 3.4.07.045) and Zurich Lung League (unrestricted grant). There was no involvement of these funding sources in study design; in the collection, analysis or interpretation of data; in the writing of the report; or in the decision to submit the article for publication. The researchers are independent from the funders. Funding information for this article has been deposited with the Crossref Funder Registry.

\section{References}

1 Vogelmeier CF, Criner GJ, Martinez FJ, et al. Global Strategy for the Diagnosis, Management, and Prevention of Chronic Obstructive Lung Disease 2017 Report. GOLD Executive Summary. Am J Respir Crit Care Med 2017; 195: 557-582.

2 Miravitlles M, Anzueto A, Legnani D, et al. Patient's perception of exacerbations of COPD - the PERCEIVE study. Respir Med 2007; 101: 453-460.

3 Pitta F, Troosters T, Spruit MA, et al. Characteristics of physical activities in daily life in chronic obstructive pulmonary disease. Am J Respir Crit Care Med 2005; 171: 972-977.

4 Hartman JE, Boezen HM, de Greef MHG, et al. Consequences of physical inactivity in chronic obstructive pulmonary disease. Expert Rev Respir Med 2010; 4: 735-745.

5 Cooper CB. Exercise in chronic pulmonary disease: limitations and rehabilitation. Med Sci Sports Exerc 2001; 33: S643-S646.

6 Breyer M, Breyer-Kohansal R, Funk G, et al. Nordic walking improves daily physical activities in COPD: a randomised controlled trial. Respir Res 2010; 22: 1-9.

7 Reardon JZ, Lareau SC, ZuWallack R. Functional status and quality of life in chronic obstructive pulmonary disease. Am J Med 2006; 119: S32-S37.

8 Polkey MI, Moxham J. Attacking the disease spiral in chronic obstructive pulmonary disease. Clin Med 2006; 6: 190-196.

9 Decramer M, Rennard S, Troosters T, et al. COPD as a lung disease with systemic consequences - clinical impact, mechanisms, and potential for early intervention. COPD 2008; 5: 235-256.

10 Earp JA, Ennett ST. Conceptual models for health education research and practice. Health Educ Res 1991; 6: 163-171.

11 Centre for Reviews and Dissemination. Systematic reviews. CRD's guidance for undertaking reviews in health care. 2009. www.york.ac.uk/inst/crd/SysRev/!SSL!/WebHelp/SysRev3.htm Date last accessed: May 31, 2017.

12 Higgins JPT, Green S, eds. Cochrane Handbook for Systematic Reviews of Interventions. Version 5.0.2. London, Cochrane, 2009.

13 Liberati A, Altman DG, Tetzlaff J, et al. The PRISMA statement for reporting systematic reviews and meta-analyses of studies that evaluate health care interventions: explanation and elaboration. Ann Intern Med 2009; 151: W65-W94.

14 Garcia-Aymerich J, Gómez FP, Benet M, et al. Identification and prospective validation of clinically relevant chronic obstructive pulmonary disease (COPD) subtypes. Thorax 2011; 66: 430-437. 
15 Strassmann A, Frei A, Haile SR, et al. Commonly used patient-reported outcomes do not improve prediction of COPD exacerbations: a multicenter 41/2 year prospective cohort study. Chest 2017; 152: 1179-1187.

16 Bestall JC, Paul EA, Garrod R, et al. Usefulness of the Medical Research Council (MRC) dyspnoea scale as a measure of disability in patients with chronic obstructive pulmonary disease. Thorax 1999; 54: 581-586.

17 Dipietro L, Caspersen CJ, Ostfeld AM, et al. A survey for assessing physical activity among older adults. Med Sci Sports Exerc 1993; 25: 628-642.

18 Donaire-Gonzalez D, Gimeno-Santos E, Serra I, et al. Validation of the Yale Physical Activity Survey in chronic obstructive pulmonary disease patients. Arch Bronconeumol 2011; 47: 552-560.

19 Siebeling L, Wiebers S, Beem L, et al. Validity and reproducibility of a physical activity questionnaire for older adults: questionnaire versus accelerometer for assessing physical activity. Clin Epidemiol 2012; 4: 171-180.

20 Puhan MA, Siebeling L, Zoller M, et al. Simple functional performance tests and mortality in COPD. Eur Respir J 2013; 42: 956-963.

21 Casanova C, Cote C, de Torres JP, et al. Inspiratory-to-total lung capacity ratio predicts mortality in patients with chronic obstructive pulmonary disease. Am J Respir Crit Care Med 2005; 171: 591-597.

22 Garcia-Aymerich J, Gómez FP, Antó JM. Caracterización fenotípica y evolución de la EPOC en el estudio PAC-COPD: diseño y metodología. [Phenotypic characterization and course of chronic obstructive pulmonary disease in the PAC-COPD study: design and methods.] Arch Bronconeumol 2009; 45: 4-11.

23 Siebeling L, ter Riet G, van der Wal WM, et al. ICE COLD ERIC - International collaborative effort on chronic obstructive lung disease: exacerbation risk index cohorts - study protocol for an international COPD cohort study. BMC Pulm Med 2009; 9: 15.

24 MacCallum RC, Browne MW, Sugawara HM. Power analysis and determination of sample size for covariance structure modeling. Psychol Methods 1996; 1: 130-149.

25 Bollen K. Structural equation models. In: Armitage P, Colton T, eds. Encyclopedia of Biostatistics. New York, Wiley, 1998; pp. 4363-4372.

26 Hooper D, Coughlan J, Mullen MR. Structural equation modelling: guidelines for determining model fit. Electron J Business Res Methods 2008; 6: 53-60.

27 Tabachnick BG, Fidell LS. Using Multivariate Statistics. 5th Edn. New York, Allyn \& Bacon, 2007.

28 Browne MW, Cudeck R. Alternative ways of assessing model fit. Sociol Methods Res 1992; 21: 123-131.

29 Hu LT, Bentler PM. Cutoff criteria for fit indexes in covariance structure analysis: conventional criteria versus new alternatives. Struct Equation Model 1999; 6: 1-55.

30 Altenburg WA, Bossenbroek L, de Greef MHG, et al. Functional and psychological variables both affect daily physical activity in COPD: a structural equations model. Respir Med 2013; 107: 1740-1747.

31 Dubow EF, Boxer P, Huesmann LR. Long-term effects of parents' education on children's educational and occupational success: mediation by family interactions, child aggression, and teenage aspirations. Merrill Palmer $Q$ 2009; 55: 224-249.

32 Cooper CB. The connection between chronic obstructive pulmonary disease symptoms and hyperinflation and its impact on exercise and function. Am J Med 2006; 119: 21-31.

33 Decramer M. Tiotropium as essential maintenance therapy in COPD. Eur Respir Rev 2006; 15: 51-57.

34 Donaldson AV, Maddocks M, Martolini D, et al. Muscle function in COPD: a complex interplay. Int J COPD 2012; 7: 523-535.

35 Maltais F. Exercise and COPD: therapeutic responses, disease-related outcomes, and activity-promotion strategies. Phys Sportsmed 2013; 41: 66-80.

36 Garcia-Aymerich J, Pitta F. Promoting regular physical activity in pulmonary rehabilitation. Clin Chest Med 2014; 35: $363-368$.

37 Corhay JL, Dang DN, Van Cauwenberge H, et al. Pulmonary rehabilitation and COPD: providing patients a good environment for optimizing therapy. Int J Chron Obstruct Pulmon Dis 2014; 9: 27-39.

38 Celli BR, MacNee W. Standards for the diagnosis and treatment of patients with COPD: a summary of the ATS/ ERS position paper. Eur Respir J 2004; 23: 932-946.

39 O'Donnell DE, Guenette JA, Maltais F, et al. Decline of resting inspiratory capacity in COPD: the impact on breathing pattern, dyspnea, and ventilatory capacity during exercise. Chest 2012; 141: 753-762.

40 Marin JM, Carrizo SJ, Gascon M, et al. Inspiratory capacity, dynamic hyperinflation, breathlessness, and exercise performance during the 6-minute-walk test in chronic obstructive pulmonary disease. Am J Respir Crit Care Med 2001; 163: 1395-1399.

41 Ramon MA, Ferrer J, Gimeno-Santos E, et al. Inspiratory capacity-to-total lung capacity ratio and dyspnoea predict exercise capacity decline in COPD. Respirology 2016; 21: 476-482.

42 Ramon MA, Gimeno-Santos E, Ferrer J, et al. Hospital admissions and exercise capacity decline in patients with COPD. Eur Respir J 2014; 43: 1018-1027.

43 Goldstein RS, Hill K, Brooks D, et al. Pulmonary rehabilitation. A review of the recent literature. Chest 2014; 142: 738-749. 